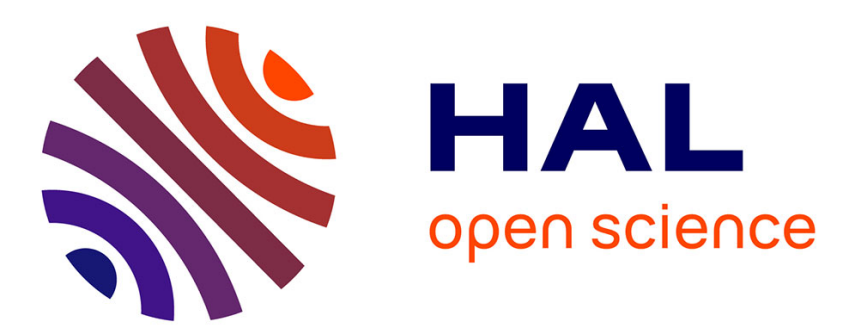

\title{
Homogenized thermal conduction model for particulate foods
}

Francisco Chinesta, Rafael Torres, Antonio Ramón, Mari Carmen Rodrigo, Miguel Rodrigo

\section{- To cite this version:}

Francisco Chinesta, Rafael Torres, Antonio Ramón, Mari Carmen Rodrigo, Miguel Rodrigo. Homogenized thermal conduction model for particulate foods. International Journal of Thermal Sciences, 2002, 41, pp.1141-1150. 10.1016/S1290-0729(02)01400-X . hal-00020718

\section{HAL Id: hal-00020718 https://hal.science/hal-00020718}

Submitted on 16 Mar 2018

HAL is a multi-disciplinary open access archive for the deposit and dissemination of scientific research documents, whether they are published or not. The documents may come from teaching and research institutions in France or abroad, or from public or private research centers.
L'archive ouverte pluridisciplinaire HAL, est destinée au dépôt et à la diffusion de documents scientifiques de niveau recherche, publiés ou non, émanant des établissements d'enseignement et de recherche français ou étrangers, des laboratoires publics ou privés. 


\title{
Homogenized thermal conduction model for particulate foods
}

\author{
Francisco Chinesta $^{\mathrm{a}, *}$, Rafael Torres ${ }^{\mathrm{b}}$, Antonio Ramon ${ }^{\mathrm{c}}$, Mari Carmen Rodrigo ${ }^{\mathrm{d}}$, \\ Miguel Rodrigo ${ }^{\mathrm{d}}$ \\ a Laboratoire de mécanique des systèmes et des procédés, École nationale supérieure d'arts et métiers, \\ 151 boulevard de l'Hôpital, 75013 Paris, France \\ b Departamento de Ingeniería Mecánica, Universidad Politécnica de Valencia, Camino de Vera s/n. 46071 Valencia, Spain \\ c AIMPLAS, Gustave Eiffel 4, 46980 Paterna, Valencia, Spain \\ d Instituto de Agroquímica y Tecnología de Alimentos, Consejo Superior de Investigaciones Científicas, \\ Apartado de correos 73, 46100 Burjasot, Spain
}

\begin{abstract}
This paper deals with the definition of an equivalent thermal conductivity for particulate foods. An homogenized thermal model is used to asses the effect of particulate spatial distribution and differences in thermal conductivities. We prove that the spatial average of the conductivity can be used in an homogenized heat transfer model if the conductivity differences among the food components are not very large, usually the highest conductivity ratio between the foods components is lower than 5 . In the general case we propose to use a standard spatial homogenisation procedure. Although the heterogeneity give rise to an anisotropic heat transfer behaviour, this effect is negligible when the food particles are randomly distributed. When we use pre-mixed particulate foods a statistical average can be defined from a small number of possible particle arrangements.
\end{abstract}

\section{Introduction}

In the food industry, thermal sterilization of packaged particulate foods is common. This is the case of vegetable salads, for example. During this process, the product placed in a pack is subjected to a heating cycle. The sterilization process requires that the lethality of heat received by all points in the package is adequate for safety. Since the kinetics of microorganisms destruction depends on both time and temperature, we must know the temperature-history at any point of the product during the process to be able to predict the lethal effects of heat. On the other hand overprocessing may produce a reduction on the food volume production as well as damage its quality. A thermal overtreatment can affect unfavourably the sensorial properties

\footnotetext{
* Corresponding author.

E-mail address: francisco.chinesta@paris.ensam.fr (F. Chinesta).
}

(texture, taste, ...), colour, amount of vitamins, ..., of the food.

Numerical simulation can be used to optimize heat treatments to reach level of microbiological lethality. Developing of an appropriate and accurate simulation algorithm is important for the food industry.

Thermal analysis of homogeneous foods is easy; the temperature evolution can be calculated from the solution of the heat transfer model [1], defined by

$\rho C \frac{\partial T}{\partial t}+\operatorname{div} q=0 \quad$ in $\Omega$

where $\Omega$ represents the pack volume; temperature $T$ depends on the space and time variables $T(\boldsymbol{x}, t), C$ is the specific heat and $\boldsymbol{q}$ the heat flux vector given by Fourier's law

$\boldsymbol{q}=-\boldsymbol{k} \operatorname{Grad} T$

where $\boldsymbol{k}$ is the conductivity tensor, which is diagonal in an isotropic medium (the heat flux does not depend on the direction considered). In the anisotropic case the tensor $\boldsymbol{k}$ 


\begin{tabular}{|c|c|c|c|}
\hline \multicolumn{4}{|c|}{ Nomenclature } \\
\hline$C$ & specific heat . & $q$ & heat flux... \\
\hline $\operatorname{div}$ & divergence operator & $Q$ & averaged heat flux... \\
\hline$D_{T}$ & reduction time in inactivation kinetics at & $R$ & gas constant.. \\
\hline$D_{R T}$ & $\begin{array}{l}\text { temperature } T \ldots \ldots \ldots \ldots \ldots \\
\text { reduction time at the reference }\end{array}$ & $t$ & time $\ldots \ldots \ldots$ \\
\hline & temperature ${ }^{1} \ldots \ldots \ldots \ldots \ldots \ldots$ & $T$ & temperature field $\ldots \ldots \ldots \ldots \ldots \ldots \ldots \ldots, \mathrm{K}$ \\
\hline$E_{a}$ & activation energy ............ & $I_{R}$ & $\begin{array}{l}\text { reference temperature in microorganisms } \\
\text { inactivation kinetics }{ }^{1} \ldots \ldots \ldots \ldots \ldots \ldots \ldots, \mathrm{K}\end{array}$ \\
\hline$F_{T}^{z}$ & lethality ${ }^{1} \ldots \ldots \ldots \ldots \ldots$ & $\operatorname{Tr}()$ & trace tensorial operator \\
\hline $\begin{array}{l}F_{0} \\
g\end{array}$ & $\begin{array}{l}\text { lethality in the coldest point }{ }^{1} \ldots \ldots \ldots \ldots \ldots, \mathrm{s} \\
\text { temperature gradient } \ldots \ldots \ldots \ldots \ldots \ldots \mathrm{K} \cdot \mathrm{m}^{-1}\end{array}$ & $x$ & $\begin{array}{l}\text { spatial coordinates. From Section } 2 \text { this symbol } \\
\text { indicates the coordinates of a point into the }\end{array}$ \\
\hline $\begin{array}{l}G \\
\operatorname{grad}\end{array}$ & $\begin{array}{l}\text { averaged temperature gradient } \ldots \ldots \ldots, \mathrm{K} \cdot \mathrm{m}^{-1} \\
\text { gradient operator }\end{array}$ & $\boldsymbol{X}$ & $\begin{array}{l}\text { characteristic volume } \Omega_{r} \ldots \ldots \ldots \ldots \ldots \ldots \mathrm{m} \\
\text { vector to locate the characteristic volumes } \Omega_{r} \text { in }\end{array}$ \\
\hline $\begin{array}{l}I \\
k\end{array}$ & $\begin{array}{l}\text { unit tensor } \\
\text { thermal conductivity tensor } \ldots \ldots \mathrm{W} \cdot \mathrm{m}^{-1} \cdot \mathrm{K}^{-1}\end{array}$ & & the global domain $\Omega$. This represents also the \\
\hline$K$ & $\begin{array}{l}\text { homogenized thermal conductivity } \\
\text { tensor } \ldots \ldots \ldots \ldots \ldots \ldots \ldots \ldots, \mathrm{W} \cdot \mathrm{m}^{-1} \cdot \mathrm{K}^{-1}\end{array}$ & & $\begin{array}{l}\text { spatial coordinates in the homogenized thermal } \\
\text { model } \ldots \ldots \ldots \ldots \ldots \ldots \ldots \ldots \ldots \ldots \ldots\end{array}$ \\
\hline$K_{T}$ & $\begin{array}{l}\text { velocity constant in the microorganisms } \\
\text { inactivation kinetics }{ }^{1} \ldots \ldots \ldots \ldots \ldots \ldots \ldots \mathrm{s}^{-1}\end{array}$ & $z$ & $\begin{array}{l}\text { temperature constant in inactivation } \\
\text { kinetics }{ }^{1} \ldots \ldots \ldots \ldots \ldots \ldots \ldots \ldots \ldots \ldots, \mathrm{K}\end{array}$ \\
\hline$K_{R T}$ & velocity constant at the reference & $\lambda_{j}$ & tensor eigenvalue \\
\hline & temperature $^{1} \ldots \ldots \ldots \ldots \ldots \ldots$ & $\rho$ & density........... \\
\hline $\boldsymbol{n}$ & outwards unit vector & $\Omega$ & domain volume ... \\
\hline$N$ & number of microorganisms & $\Omega_{r}$ & characteristic volume \\
\hline$N_{0}$ & initial number of microorganisms & $\partial \Omega$ & domain boundary $\ldots \ldots \ldots \ldots \ldots \ldots \ldots \ldots \ldots \mathrm{m}^{2}$ \\
\hline
\end{tabular}

is also diagonal when the system of coordinates associated with the eigenvectors of $\boldsymbol{k}$ is considered. Moreover, due to the homogeneity assumption, $\boldsymbol{k}$ is independent of the spatial coordinates.

Initial condition

$T(\boldsymbol{x}, t=0)=T_{0}(\boldsymbol{x})$

may be considered constant, i.e., $T_{0}(\boldsymbol{x})=T_{0}$.

The boundary condition is

$T(x \in \partial \Omega, t)=T_{d}(t)$

where $\partial \Omega$ represents the surface of the pack domain $\Omega$, and $T_{d}(t)$ the surface temperature at time $t$.

This differential equation (1) can be solved using any of several numerical strategies; e.g., the finite element method, the finite difference method, the spectral techniques, the boundary element method, etc.

However, both the heat transfer model and its numerical simulation are difficult to apply if the medium is heterogeneous (particulate food), with a characteristic length (particles size) lower than the associated with the global thermal problem (characteristic length of the food pack). Then we designate by particle and product scales the ones associated respectively with the conductivity variation (characteristic length of the particles) and to the domain geometry.

\footnotetext{
${ }^{1}$ In food science and technology applications $K_{T}$ and $K_{R T}$ are usually expressed in $\min ^{-1} ; D_{T}, D_{R T}, F_{T}^{z}$ and $F_{0}$ in min and $T, T_{R}$ and $z$ in ${ }^{\circ} \mathrm{C}$.
}

When a system is heterogeneous $\rho, C$ and $\boldsymbol{k}$ depend on the spatial coordinates

$\rho C(\boldsymbol{x}) \frac{\partial T}{\partial t}+\operatorname{div}(-\boldsymbol{k}(\boldsymbol{x}) \operatorname{grad} T)=0$

A standard discretisation technique for solving the heat transfer model (5) should consider a mesh size of the same order of magnitude as the characteristic length of the source of heterogeneity, i.e., the size of the particles. This introduces a strong handicap in the efficiency of the numerical discretisation techniques, specially for threedimensional heterogeneous cases.

The definition of an equivalent heat transfer model, which operates in a locally homogeneous equivalent medium is of major interest in order to obtain sterilization predictions in real particulate foods. As the heat transfer model (5) implies conductivity derivatives, the usual spatial average techniques are not a correct way to obtain these equivalent models.

By assuming a periodic arrangement of the food particles, an homogeneous equivalent heat transfer model can be defined by using a multiple scale technique (by means of the asymptotic expansions of the temperature variable) [2].

In this work we use a general technique to obtain the scale transfer tensor, by solving three elliptic boundary value problems, in order to obtain the equivalent conductivity in each region of $\Omega$ where the volume fraction of each food component is constant [3-7]. As a consequence of this homogenisation process, we obtain an anisotropic equivalent conductivity, although each food component has its own 
isotropic conductivity. In the same way, the food particle distribution in the pack is not known a priori, so a statistical average is required to describe the different particulate arrangements. Finally, a three-dimensional Galerkin formulation in finite elements is used in the numerical simulation of the industrial processes involving particulate foods.

\section{Homogenized thermal model}

\subsection{Spatial homogenisation}

We will consider a characteristic volume $\Omega_{r}$, very small compared to the domain size. $\Omega_{r}$ contains enough particles to offer a good representation of each food component.

Due to possible variation of the volume fraction of some food component or of the food particles arrangement in the global volume, we need to locate $\Omega_{r}$ in $\Omega$, and for this purpose the notation $\Omega_{r}(\boldsymbol{X})$ is used.

From now on, we call particle scale, the scale associated with $\Omega_{r}$, and product scale, the scale related to the global domain $\Omega$. So, the variation of the variables inside $\Omega_{r}$ will be described only by a local analysis in $\Omega_{r}$. The variations in $\Omega$ require a global analysis, which does not enable to obtain information about the temperature evolution in the scale associated with the particles. Capital letters will be used to denote the variables at the product scale.

Obviously, at the particle scale, the thermal behaviour is perfectly defined by the Fourier's law

$q(x)=-k(x) g(x)$

where $\boldsymbol{g}(\boldsymbol{x})$ represents the temperature gradient at the point $\boldsymbol{x} \in \Omega_{r}(\boldsymbol{X})$.

Our main aim will be to determine an equivalent relation in the scale of the product in the form

$Q(X)=-K(X) G(X)$

with

$\left\{\begin{array}{l}\boldsymbol{Q}(\boldsymbol{X})=\left\langle\left.\boldsymbol{q}(\boldsymbol{x})\right|_{\Omega_{r}(\boldsymbol{X})}\right. \\ \boldsymbol{G}(\boldsymbol{X})=\langle\boldsymbol{g}(\boldsymbol{x})\rangle_{\Omega_{r}(\boldsymbol{X})}\end{array}\right.$

where the spatial averaging of a generic vectorial field $\boldsymbol{a}(\boldsymbol{x}),\langle\boldsymbol{a}\rangle$, is given by

$\langle\boldsymbol{a}(\boldsymbol{x})\rangle_{\Omega_{r}(\boldsymbol{X})}=\frac{1}{\left|\Omega_{r}(\boldsymbol{X})\right|} \int_{\Omega_{r}(\boldsymbol{X})} \boldsymbol{a}(\boldsymbol{x}) \mathrm{d} \Omega$

and where $\left|\Omega_{r}(\boldsymbol{X})\right|$ represents the volume of $\Omega_{r}(\boldsymbol{X})$.

We introduce the tensor $\boldsymbol{M}(\boldsymbol{x}, \boldsymbol{X})$, defined in each point $\boldsymbol{x} \in \Omega_{r}(\boldsymbol{X})$ to establish a relation between both scales

$\boldsymbol{g}(\boldsymbol{x})=\boldsymbol{M}(\boldsymbol{x}, \boldsymbol{X}) \boldsymbol{G}(\boldsymbol{X})$

The homogenisation results in

$$
\begin{aligned}
\boldsymbol{Q} & =\langle\boldsymbol{q}\rangle_{\Omega_{r}(\boldsymbol{X})}=-\langle\boldsymbol{k} \boldsymbol{g}\rangle_{\Omega_{r}(\boldsymbol{X})}=-\langle\boldsymbol{k} \boldsymbol{M} \boldsymbol{G}\rangle_{\Omega_{r}(\boldsymbol{X})} \\
& =-\langle\boldsymbol{k} \boldsymbol{M}\rangle_{\Omega_{r}(\boldsymbol{X})} \boldsymbol{G}=-\boldsymbol{K} \boldsymbol{G}
\end{aligned}
$$

Thus, with $\boldsymbol{M}(\boldsymbol{x}, \boldsymbol{X})$ known, it is possible to obtain the equivalent conductivity tensor $\boldsymbol{K}(\boldsymbol{X})$, which is defined in $\Omega$, and whose variations are due only to the possible nonuniform distribution of the volume fraction of each food component in $\Omega$; however the tensor $\boldsymbol{K}(\boldsymbol{X})$ does not depend explicitly on the conductivity heterogeneity associated with particles.

If we are able to obtain $\boldsymbol{M}(\boldsymbol{x}, \boldsymbol{X})$, the previous procedure allows us to obtain $\boldsymbol{K}(\boldsymbol{X})$, and to write the equivalent heat transfer equation

$\rho C(\boldsymbol{X}) \frac{\partial T}{\partial t}+\operatorname{div}_{\boldsymbol{X}}\left(-\boldsymbol{K}(\boldsymbol{X}) \operatorname{grad}_{\boldsymbol{X}} T\right)=0$

where $\operatorname{div}_{\boldsymbol{X}}$ and $\operatorname{grad}_{\boldsymbol{X}}$ represent the differential operators related to the product scale, and where the equivalent specific heat is obtained by direct spatial average because this parameter is not affected by any differential operator.

\subsection{The scale transfer tensor}

Let's the definition of the scale transfer tensor

$g(x)=M(x, X) G(X)$

Now, we solve three steady state heat transfer problems in $\Omega_{r}(\boldsymbol{X})$, with an average temperature gradient imposed in each one

$$
\begin{aligned}
& \boldsymbol{G}^{1}(\boldsymbol{X})=\left(\begin{array}{l}
1 \\
0 \\
0
\end{array}\right), \quad \boldsymbol{G}^{2}(\boldsymbol{X})=\left(\begin{array}{l}
0 \\
1 \\
0
\end{array}\right) \\
& \boldsymbol{G}^{3}(\boldsymbol{X})=\left(\begin{array}{l}
0 \\
0 \\
1
\end{array}\right)
\end{aligned}
$$

The temperature field associated with the solution of the steady heat transfer problem

$\operatorname{div}(-\boldsymbol{k}(\boldsymbol{x}) \operatorname{grad} T)=0$

in $\Omega_{r}(\boldsymbol{X})$, with the condition $\boldsymbol{G}^{i}(\boldsymbol{X})$ will be denoted by $T^{i}(\boldsymbol{x})$. With these temperature fields obtained we can compute their gradients obtaining the vector fields $g^{1}(x), g^{2}(x)$ and $\boldsymbol{g}^{3}(\boldsymbol{x})$ respectively, i.e., the temperature gradient at each point $\boldsymbol{x} \in \Omega_{r}(\boldsymbol{X})$. Thus, obviously, at any point $\boldsymbol{x} \in \Omega_{r}(\boldsymbol{X})$, the first column of $\boldsymbol{M}(\boldsymbol{x}, \boldsymbol{X})$ corresponds to the vector $g^{1}(x)$, the second one to $g^{2}(x)$, and the last one to $g^{3}(x)$.

If we solve these three steady state heat problems, the scale transfer tensor is fully determined. However, the heat transfer model imposing an average temperature gradient, defines an ill-posed problem. Moreover, the average temperature gradient condition may be transformed in an homogeneous boundary condition [6]

$T\left(\boldsymbol{x} \in \partial \Omega_{r}(\boldsymbol{X})\right)=\boldsymbol{G}^{\mathrm{T}} \boldsymbol{x}$

where $\boldsymbol{G}^{\mathrm{T}}$ represents the transposed vector of $\boldsymbol{G}$.

In this form the steady heat transfer problems are defined by

$\operatorname{div}\left(-\boldsymbol{k}(\boldsymbol{x}) \operatorname{grad} T^{i}(\boldsymbol{x})\right)=0$ 
in $\Omega_{r}(\boldsymbol{X})$, with $T^{i}\left(\boldsymbol{x} \in \partial \Omega_{r}(\boldsymbol{X})\right)=\boldsymbol{G}^{i^{\mathrm{T}}} \boldsymbol{x}, i=1,2,3$; which now results in three well defined boundary value problems that can be solved by using a standard formulation of finite elements.

We can verify that both conditions (14) and (16) are equivalent:

$$
\begin{aligned}
\boldsymbol{G}(\boldsymbol{X}) & =\langle\boldsymbol{g}(\boldsymbol{x})\rangle_{\Omega_{r}(\boldsymbol{X})}=\frac{1}{\left|\Omega_{r}(\boldsymbol{X})\right|} \int_{\Omega_{r}(\boldsymbol{X})} \boldsymbol{g}(\boldsymbol{x}) \mathrm{d} \Omega \\
& =\frac{1}{\left|\Omega_{r}(\boldsymbol{X})\right|} \int_{\Omega_{r}(\boldsymbol{X})} \operatorname{grad}_{\boldsymbol{x}} T \mathrm{~d} \Omega \\
& =\frac{1}{\left|\Omega_{r}(\boldsymbol{X})\right|} \int_{\partial \Omega_{r}(\boldsymbol{X})} T \boldsymbol{n} \mathrm{d} S \\
& =\frac{1}{\left|\Omega_{r}(\boldsymbol{X})\right|} \int_{\partial \Omega_{r}(\boldsymbol{X})}\left(\boldsymbol{G}^{\mathrm{T}} \boldsymbol{x}\right) \boldsymbol{n} \mathrm{d} S \\
& =\frac{1}{\left|\Omega_{r}(\boldsymbol{X})\right|} \int_{\Omega_{r}(\boldsymbol{X})} \operatorname{grad}_{\boldsymbol{x}}\left(\boldsymbol{G}^{\mathrm{T}} \underline{\boldsymbol{x}}\right) \mathrm{d} \Omega \\
& =\frac{\boldsymbol{G}}{\left|\Omega_{r}(\boldsymbol{X})\right|} \int_{\Omega_{r}(\boldsymbol{X})} \mathrm{d} \Omega=\boldsymbol{G}(\boldsymbol{X})
\end{aligned}
$$

\subsection{Discretisation of the heat transfer model in the representative volume}

The steady state heat transfer model is defined in the representative volume $\Omega_{r}(\boldsymbol{X})$ by

$\operatorname{div}_{\boldsymbol{x}}\left(-\boldsymbol{k}(\boldsymbol{x}) \operatorname{grad}_{\boldsymbol{x}} T\right)=0$

with the boundary condition

$T\left(\boldsymbol{x} \in \partial \Omega_{r}(\boldsymbol{X})\right)=\boldsymbol{G}^{\mathrm{T}} \boldsymbol{x}$

Due to the discontinuous conductivity in $\Omega_{r}(\boldsymbol{X})$ at the particle interfaces between different food components, we consider the variational formulation of the heat transfer model given by

Find $T(\boldsymbol{x}) \in H^{1}\left(\Omega_{r}(\boldsymbol{X})\right)$, with $T\left(\boldsymbol{x} \in \partial \Omega_{r}(\boldsymbol{X})\right)=\boldsymbol{G}^{\mathrm{T}} \boldsymbol{x}$ verifying

$$
\left\{\begin{array}{c}
\int_{\Omega_{r}(\boldsymbol{X})}\left(\operatorname{grad}_{\boldsymbol{x}} \psi\right)^{\mathrm{T}}\left(\boldsymbol{k}(\boldsymbol{x}) \operatorname{grad}_{\boldsymbol{x}} T\right) \mathrm{d} \Omega=0 \\
\forall \psi(\boldsymbol{x}) \in H_{0}^{1}\left(\Omega_{r}(\boldsymbol{X})\right)
\end{array}\right.
$$

where $H^{1}(\Omega)$ and $H_{0}^{1}(\Omega)$ are the standard Sobolev spaces [8].

This formulation can be used easily even for a discontinuous conductivity, because it does not imply a conductivity derivative. A finite element technique has been used to solve the previous model.

\subsection{Statistical average}

The real distribution of food particles in $\Omega$ is not known a priori. For example, in the food industry, a pre-mixing is usually carried out before pack filling.
Thus, to obtain the equivalent conductivity by the procedure previously proposed, we set randomly each food particle in $\Omega_{r}$. Even if all the particles are thermally isotropic, the heterogeneity in the particles distribution induces a slight anisotropic behaviour, usually lower than $1 \%$.

So, we obtain an equivalent conductivity for each food particles arrangement studied. As the real food particle distribution is unknown, we make a statistical average to take into account all the possible particles configurations.

An equivalent conductivity is found in each region with constant volume fraction of the food components, for different particles configurations $S_{i}$ inside the representative volume. From these, we can define the statistical average of the thermal conductivity

$\boldsymbol{K}(\boldsymbol{X})=\sum_{i}^{N_{S}} \boldsymbol{K}\left(\boldsymbol{X}, S_{i}\right) P\left(S_{i}\right)$

where $N_{S}$ denotes the number of possible particles arrangements, and $P\left(S_{i}\right)$ the probability associated with this configuration $S_{i}$.

If all configurations have the same probability, the statistical average of the conductivity is given by

$\boldsymbol{K}(\boldsymbol{X})=\frac{1}{N_{s}} \sum_{i}^{N_{S}} \boldsymbol{K}\left(\boldsymbol{X}, S_{i}\right)$

\subsection{The heat transfer problem defined in the food pack volume}

With the equivalent conductivity calculated from both a spatial homogenisation and a statistical average, the heat transfer problem in the pack containing the particulate food $\Omega$ can be written using the following variational formulation [8]

Find $T(X, t) \in H^{1}(\Omega), \forall t \in\left\lfloor 0, t_{f}\right\rfloor$, with $T(X \in \partial \Omega, t)$ $=T_{d}(t)$ and $T(X, t=0)=T_{0}(X)$ verifying

$$
\left\{\begin{array}{l}
\int_{\Omega} \psi \rho C(\boldsymbol{X}) \frac{\partial T}{\partial t} \mathrm{~d} \Omega \\
\quad+\int_{\Omega}\left(\operatorname{grad}_{\boldsymbol{X}} \psi\right)^{\mathrm{T}}\left(\boldsymbol{K}(\boldsymbol{X}) \operatorname{grad}_{\boldsymbol{X}} T\right) \mathrm{d} \Omega=0 \\
\quad \forall \psi(\boldsymbol{X}) \in H_{0}^{1}(\Omega), \forall t \in\left[0, t_{f}\right]
\end{array}\right.
$$

where $t_{f}$ is the thermal treatment time.

\section{Remarks.}

- When there is just one representative volume in the domain $\Omega$, the equivalent conductivity is the same for every point $\boldsymbol{X} \in \Omega$.

- An implicit or semi-implicit Euler technique may be accurately applied for the time discretization.

\section{Results and discussion}

At first we will consider an example without industrial interest, to evaluate the impact of the heterogeneity in the food distribution and the pertinence of an equivalent thermal model in relation with a spatial average of the thermal 


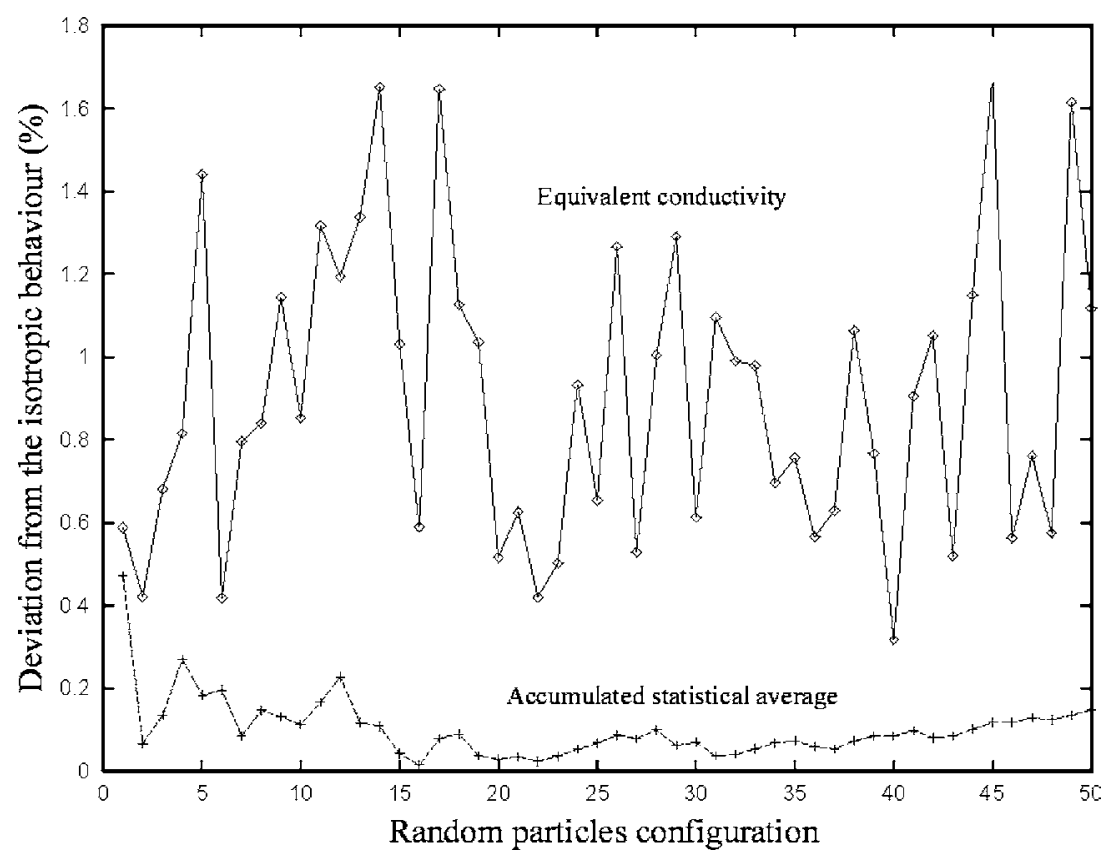

Fig. 1. Deviations from the isotropic behaviour of both the equivalent conductivity and the accumulated statistical average of the equivalent conductivity tensor for 50 random configurations.

conductivity. After that we will focus on an other example with more industrial interest, a particulate salad, which allow us to draw interesting conclusions.

We consider a cubic representative volume $\Omega_{r}$, and two food components $A$ and $B$ with the same volume fraction in order to analyse only the incidence of both the food components conductivity ratio and the particles distribution within the pack. The conductivity of each food component is isotropic, and their values are $k_{a}$ and $k_{b}$, respectively. The food particles are placed at random into the domain $\Omega_{r}$.

We define the deviation from the isotropic behaviour in $\%\left(d_{i}\right)$ of the equivalent conductivity $\boldsymbol{K}_{i}$, associated with the particles arrangement $S_{i}$, by

$d_{i}=\frac{\sqrt{\sum_{j=1}^{j=3}\left(\lambda_{j}-\operatorname{Tr}\left(\boldsymbol{K}_{i}\right) / 3\right)^{2}}}{\operatorname{Tr}\left(\boldsymbol{K}_{i}\right)} 100$

Where $\operatorname{Tr}()$ denotes the tensor operator trace, and $\lambda_{j}$ the eigenvalues of the equivalent conductivity tensor $\boldsymbol{K}_{i}$.

The accumulated statistical average of the equivalent conductivity tensor for $i$ particles arrangements, is defined as

$\boldsymbol{K}_{i}^{\text {stat }}=\frac{\sum_{j=1}^{j=i} \boldsymbol{K}_{j}}{i}$

where the same probability is considered for each particle arrangement. The deviation in \% from the isotropic behaviour for the previous tensor results

$d_{i}^{\text {stat }}=\frac{\sqrt{\sum_{j=1}^{j=3}\left(\lambda_{j}-\operatorname{Tr}\left(\boldsymbol{K}_{i}^{\text {stat }}\right) / 3\right)^{2}}}{\operatorname{Tr}\left(\boldsymbol{K}_{i}^{\text {stat }}\right)} 100$

where in this case $\lambda_{j}$ denotes the eigenvalues of $\boldsymbol{K}_{i}^{\text {stat }}$.
In Fig. 1, for 50 random particles configurations, and a conductivity ratio $k_{a} / k_{b}=5$, we show both the deviation from the isotropic behaviour for each equivalent conductivity, and the deviation from the isotropic behaviour of the accumulated statistical average (in industrial applications the highest conductivity ratio between the food components is usually lower than 5 , thus, taking the conductivity ratio in this example equal to five, we will analyse one of the most unfavourable cases). We can notice that, in all cases, the deviation for every configuration is very small (less than $1.8 \%$ ), and even with a little amount of random configurations (less than 15), the statistical average of the equivalent conductivity has a very small deviation from the isotropic behaviour (about $0.15 \%$ ). Thus, we can conclude that a small number of random configurations is sufficient to evaluate the statistical average of the conductivity.

To obtain the difference between the equivalent conductivity and the spatial average conductivity, we need to define first the spatial average as

$$
\boldsymbol{K}^{\text {spat }}=\frac{1}{\left|\Omega_{r}\right|} \int_{\Omega_{r}} \boldsymbol{k}(\boldsymbol{x}) \mathrm{d} \Omega
$$

which is in the present example results $\boldsymbol{K}^{\text {spat }}=\frac{k_{a}+k_{b}}{2} \boldsymbol{I}$ (where $\boldsymbol{I}$ is the unit tensor).

Then we define for a particle configuration $S_{i}$ (whose equivalent conductivity is $\boldsymbol{K}_{i}$ ) the deviation in $\%$ from the spatial average as

$d_{i}^{\text {spat }}=\frac{\sqrt{\sum_{j=1}^{j=3}\left(\lambda_{j}-\operatorname{Tr}\left(\boldsymbol{K}^{\text {spat }}\right) / 3\right)^{2}}}{\operatorname{Tr}\left(\boldsymbol{K}_{i}\right)} 100$

where $\lambda_{j}$ are the eigenvalues of $\boldsymbol{K}_{i}$. Fig. 2 shows this deviation, and we can notice its high values, with a mean 


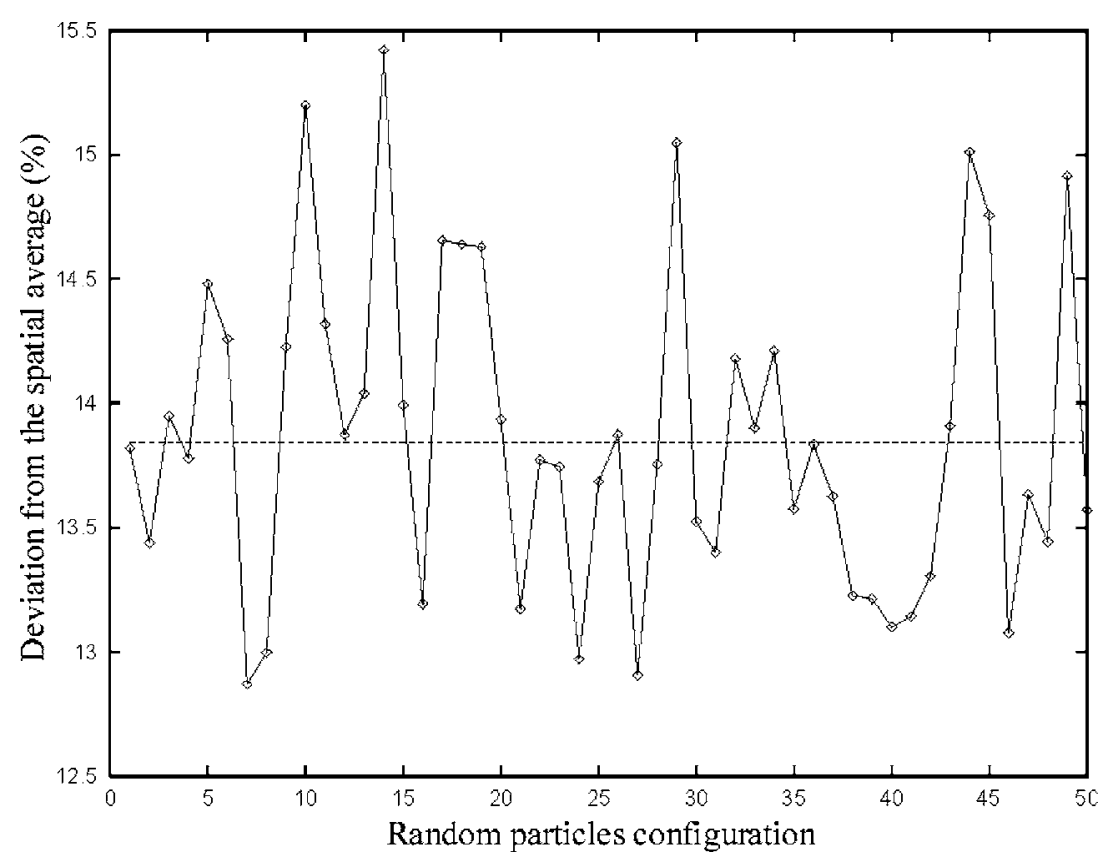

Fig. 2. Deviations from the isotropic behaviour of the spatial average conductivity for 50 random configurations.

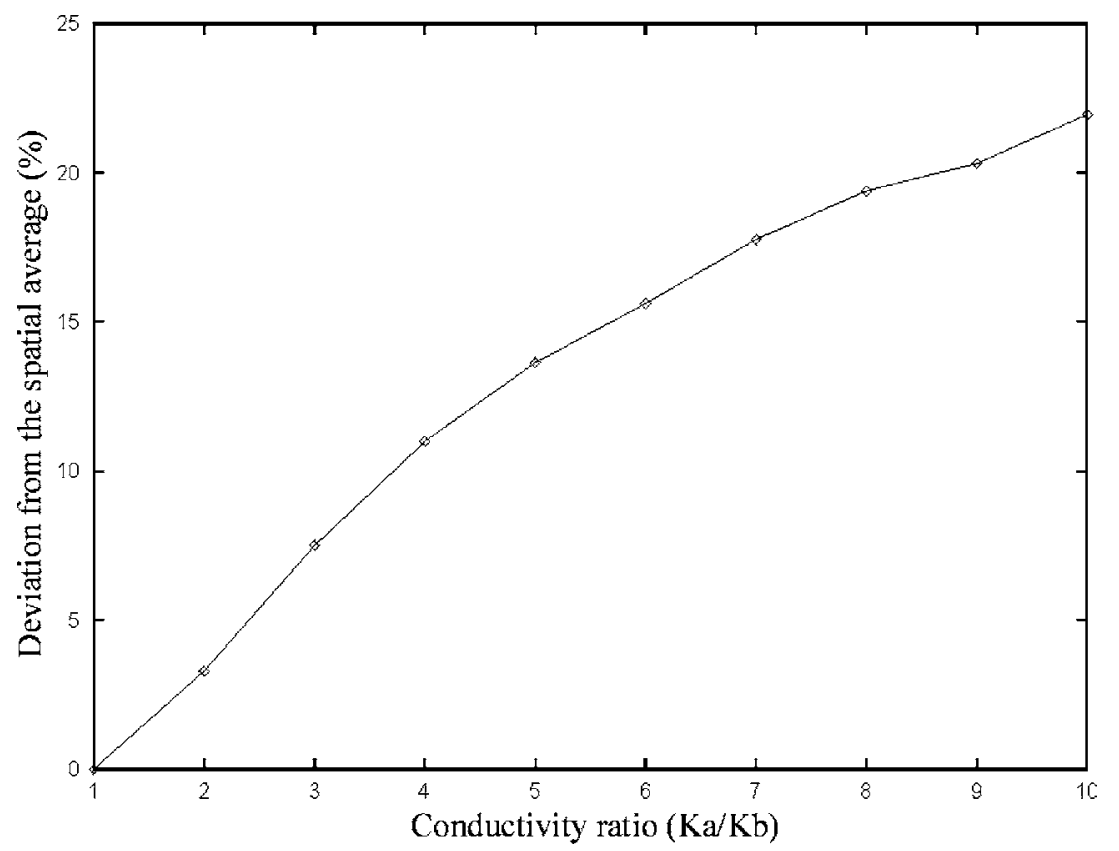

Fig. 3. Deviations from the isotropic behaviour of the spatial average for different conductivity ratios $k_{a} / k_{b}$.

value of about $14 \%$. The dependence of the deviation $d_{i}^{\text {spat }}$ on the ratio $k_{a} / k_{b}$ is depicted in Fig. 3, where the mean value of $d_{i}^{\text {spat }}$ (for 10 random particles arrangements) is represented as a function of $k_{a} / k_{b}$, with $k_{a} / k_{b} \in[1, \ldots, 10]$. From the point of view of food industries, conductivities ratios higher than 5 are not very realistic. However, we take into account conductivity ratios between 1 and 10 to illustrate a more general behaviour and to determine their limit value. We can notice that the mean value of the deviation increases with the conductivity rate $\left(k_{a} / k_{b}\right)$.
Now we consider another example with more industrial interest. This is the case of a particulate salad, composed of small pieces of tuna, corn, carrot and pepper. The composition and the thermal properties specific heat and conductivity of each ingredient $[9,10]$ are presented in Table 1 . We can notice that the conductivity differences among the different components are small. In this case the difference between the equivalent and the spatial average conductivities results lower than $0.3 \%$ for general particles arrangements. 
Table 1

Food composition and thermal properties

\begin{tabular}{lccc}
\hline Product & Mass fraction $(\%)$ & Conductivity $\left(\mathrm{W} \cdot \mathrm{m}^{-1} \cdot \mathrm{K}^{-1}\right)$ & Specific heat $\left(\mathrm{kJ} \cdot \mathrm{kg}^{-1} \cdot \mathrm{K}^{-1}\right)$ \\
\hline Tuna & 25 & 0.49 & 3.20 \\
Corn & 22 & 0.55 & 3.31 \\
Carrot & 19 & 0.55 & 3.80 \\
Pepper & 14 & 0.55 & 3.92 \\
Sauce & 10 & 0.74 & 4.25 \\
Oil & 10 & 0.18 & 1.90 \\
\hline
\end{tabular}

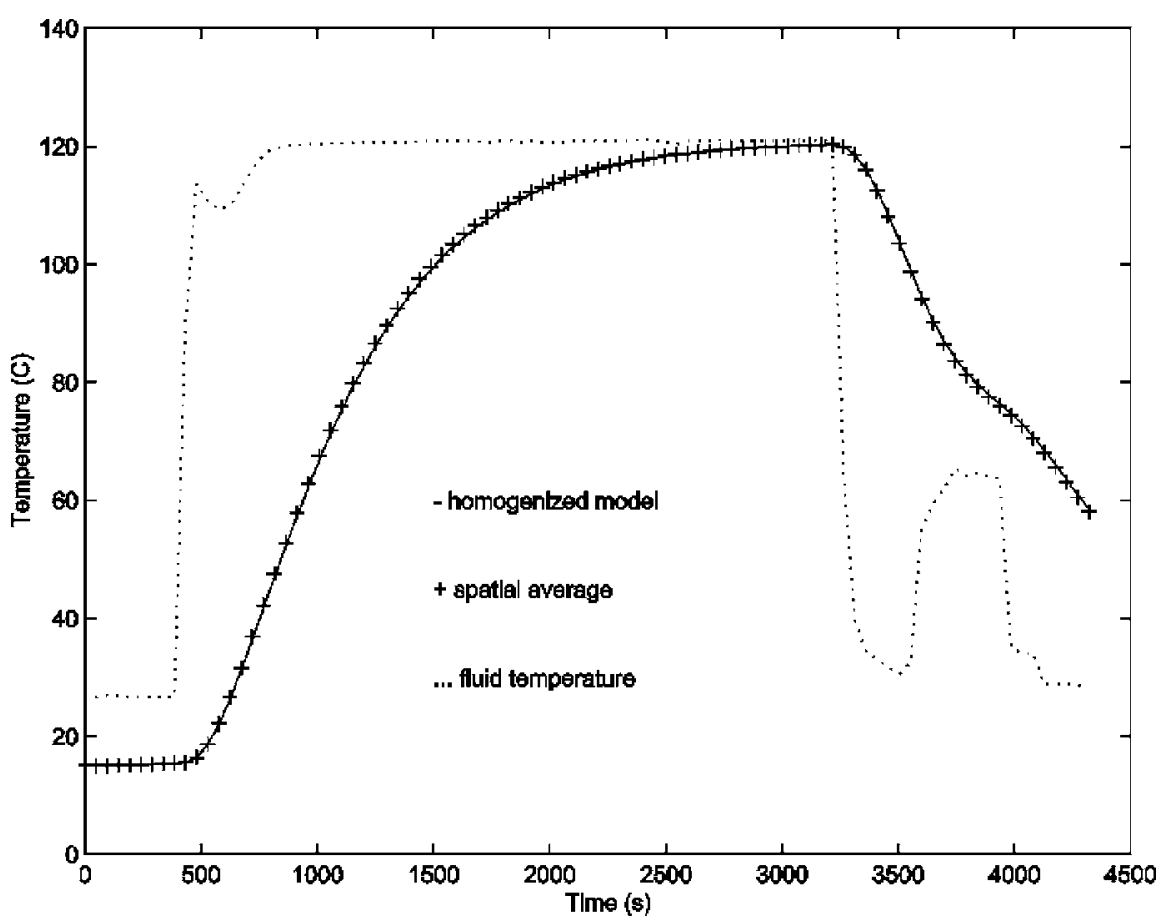

Fig. 4. Temperature evolution of the thermal fluid and predicted temperatures using equivalent and spatial average conductivities.

The previous particulate product is placed into a metallic cylindrical pack of $83.7 \mathrm{~mm}$ internal diameter and $37 \mathrm{~mm}$ height, and it is subjected to a thermal treatment. The conductivity of the metallic container is assumed infinite. The temperature evolution of the thermal fluid in contact with the food pack is depicted in Fig. 4 (dashed line). A thermocouple is placed in the middle of the height, at $9 \mathrm{~mm}$ from the axis. The temperature field at each time can be obtained solving the thermal problem defined in Section 2.5 with the equivalent conductivity resulting from the procedure described in Sections 2.1 and 2.4. Fig. 4 shows also the predicted temperatures at the point where the thermocouple was located, using both the spatial average conductivity and the equivalent conductivity obtained from spatial and statistical averages. From these results we can notice the small differences obtained from both models. Finally in Fig. 5 the predicted and the experimental measured temperatures are compared. We can notice the good agreement between both results.

The microbiological information concerning the lethality in the coldest point, the integrated lethality and the number of microorganisms can be obtained according to the proce- dure described in $[11,12]$ that we summarize in the following paragraphs.

The first order inactivation kinetics can be written as

$\frac{\mathrm{d} N}{\mathrm{~d} t}=-K_{T} N$

where $N$ represents the number of microorganisms at time $t$ and $K_{T}$ a parameter depending on the temperature $T$.

The integration of Eq. (30) results

$\ln \left(\frac{N}{N_{0}}\right)=-K_{T} t$

where $N_{0}$ is the initial number of microorganisms. The dependence of $K_{T}$ on the temperature is usually modelled by the Arrhenius equation

$K_{T}=K_{R T} e^{\left[-E_{a} / R\left(1 / T-1 / T_{R}\right)\right]}$

where $K_{R T}$ represents the value of $K$ at the reference temperature $T_{R}, E_{a}$ is the activation energy and $R$ the gas constant. 


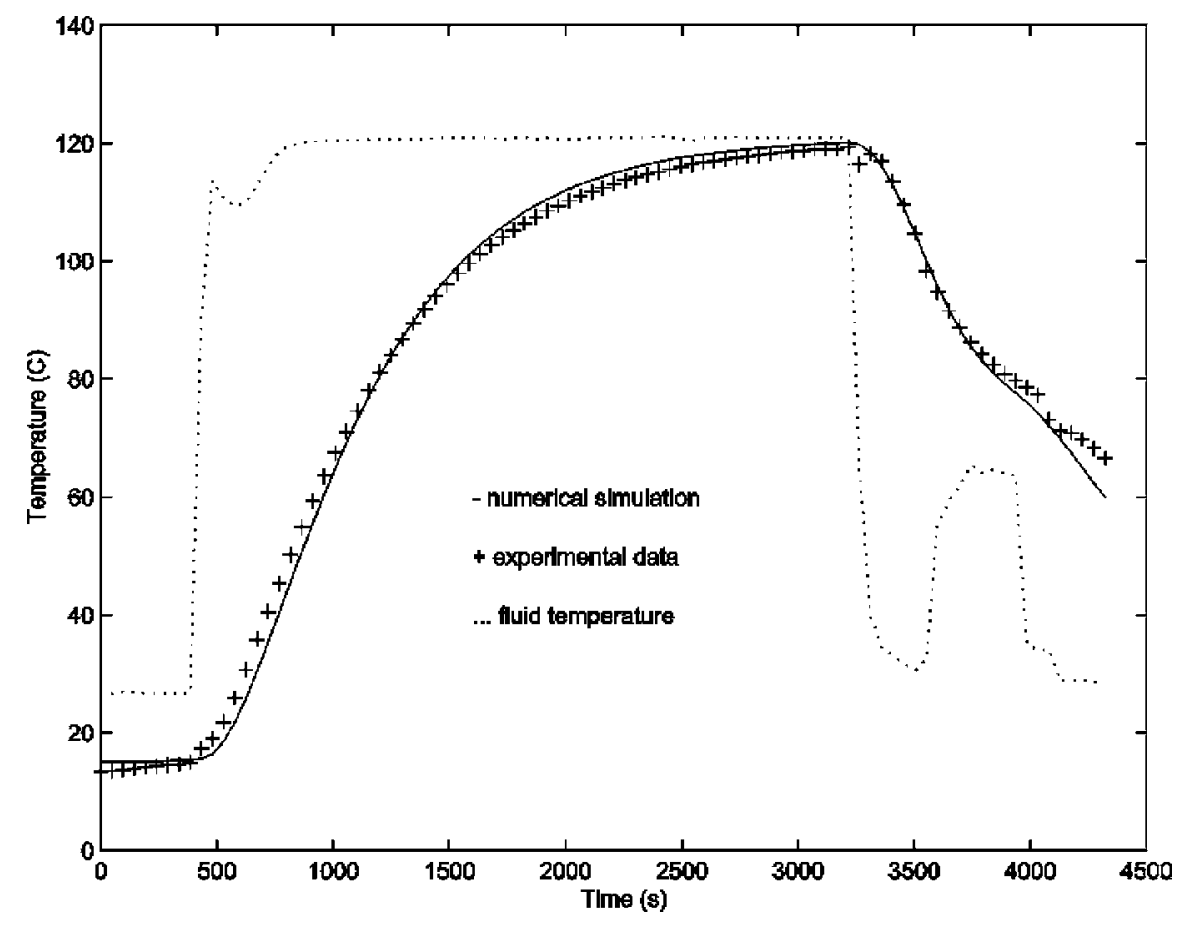

Fig. 5. Predicted versus experimental results.

On the other hand, the empirical Bigelow model establishes

$\log \left(\frac{N}{N_{0}}\right)=-\frac{1}{D_{T}} t$

where $D_{T}$ represents the process time at the temperature $T$ required to reduce by 10 the number of microorganisms. The dependence of $D_{T}$ on the temperature is assumed in the form

$\log \left(\frac{D_{T}}{D_{R T}}\right)=-\frac{1}{z}\left(T-T_{R}\right)$

where the parameter $z$ is the temperature increasing required to reduce to $10 \%$ the time $D_{R T}$.

From the Bigelow model we can obtain the expression of the microorganisms reduction during an arbitrary heating treatment

$\log \left(\frac{N}{N_{0}}\right)=\frac{1}{D_{R T}} \int_{0}^{t} 10^{\frac{T\left(t^{\prime}\right)-T_{R}}{z}} \mathrm{~d} t^{\prime}$

The lethality $F_{T}^{z}$ is defined as

$F_{T}^{z}=D_{R T} \log \left(\frac{N}{N_{0}}\right)=\int_{0}^{t} 10^{\frac{T\left(t^{\prime}\right)-T_{R}}{z}} \mathrm{~d} t^{\prime}$

Moreover, the lethality in the coldest point when $T_{R}=$ $121^{\circ} \mathrm{C}$ and $z=10^{\circ} \mathrm{C}$ is denoted by $F_{0}$

$F_{0}=\int_{0}^{t} 10^{\frac{T\left(t^{\prime}\right)-121}{10}} \mathrm{~d} t^{\prime}$

Figs. 6 and 7 show the evolution in time of both the lethality in the coldest point and the number of microorganisms for the thermal process previously described and illustrated in Fig. 4. We can notice that in spite of the slight difference in the lethality between the spatial average and the equivalent conductivity models for high times, no significant difference in the microorganism evolution is observed.

We have observed experimentally that temperatures measured at the center of the pack, placing the thermocouple in the fluid region (oil and sauce mixture) or into a food particle, are not significantly different. Moreover, the evolution of the temperature in this region is in good agreement with a heat conduction model whose equivalent conductivity, as proved, is very close to the spatial conductivities average. This fact proves that convection phenomena in the fluid phase can be neglected, because if one takes into account the heat convection, a faster temperature evolution should be obtained. The small size and the different shapes of the particles which constitutes our study system increase the tortuosity of the fluid flow paths, disturbing the natural convection. This fact allows us a temperature prediction based on the hypothesis of food components at rest. Thus, this kind of model cannot be applied to other systems where the fluid flow can be easily established, as it is the case of asparagus, seafood,... packs. In these cases experiments have shown that the evolution of the temperature in core region is faster than the numerical prediction based on an equivalent conductivity with the food components at rest. However, the modelling proposed in this paper can be successfully applied to other thermal heterogeneous systems outside the food industry. 


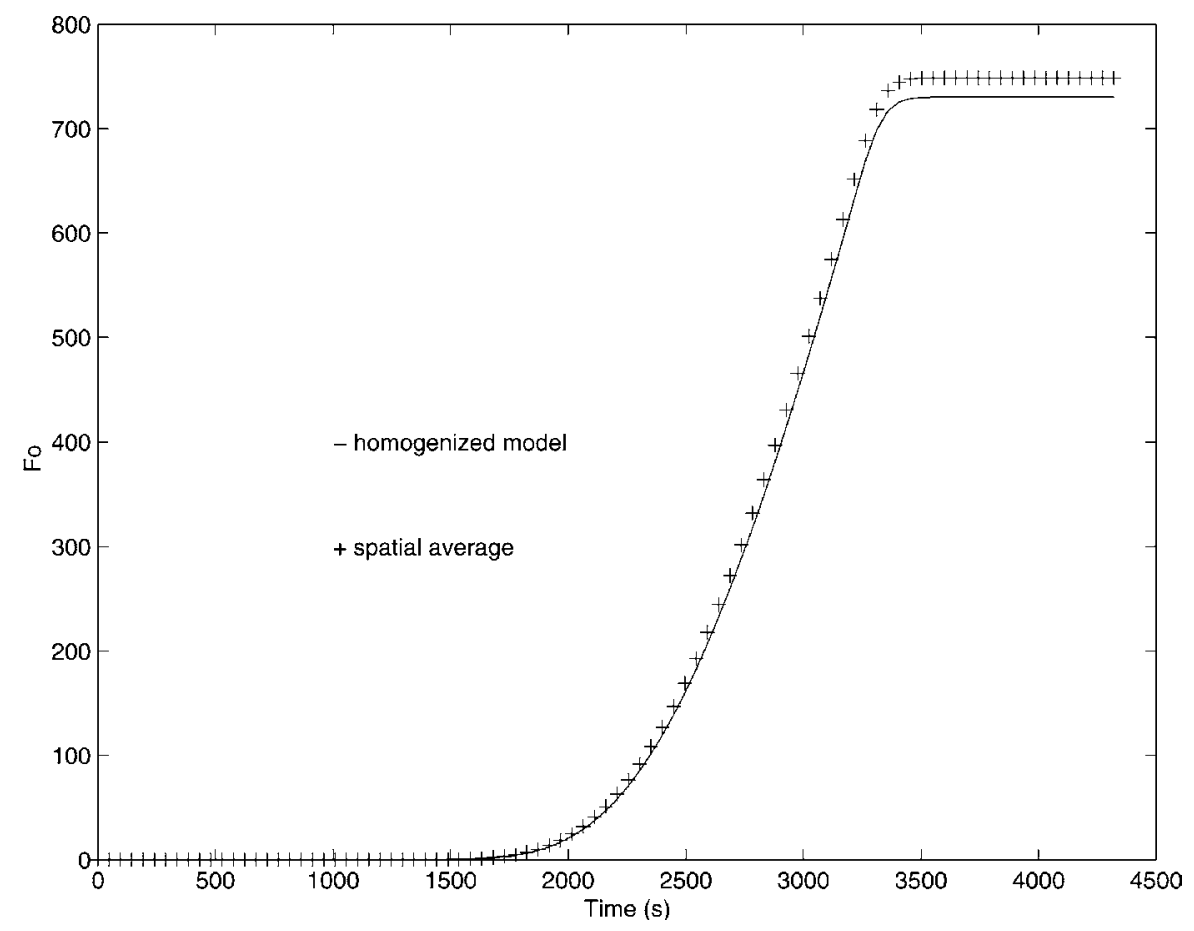

Fig. 6. Evolution in time of the lethality in the coldest point $\left(T_{R}=121^{\circ} \mathrm{C}\right.$ and $\left.z=10^{\circ} \mathrm{C}\right)$.

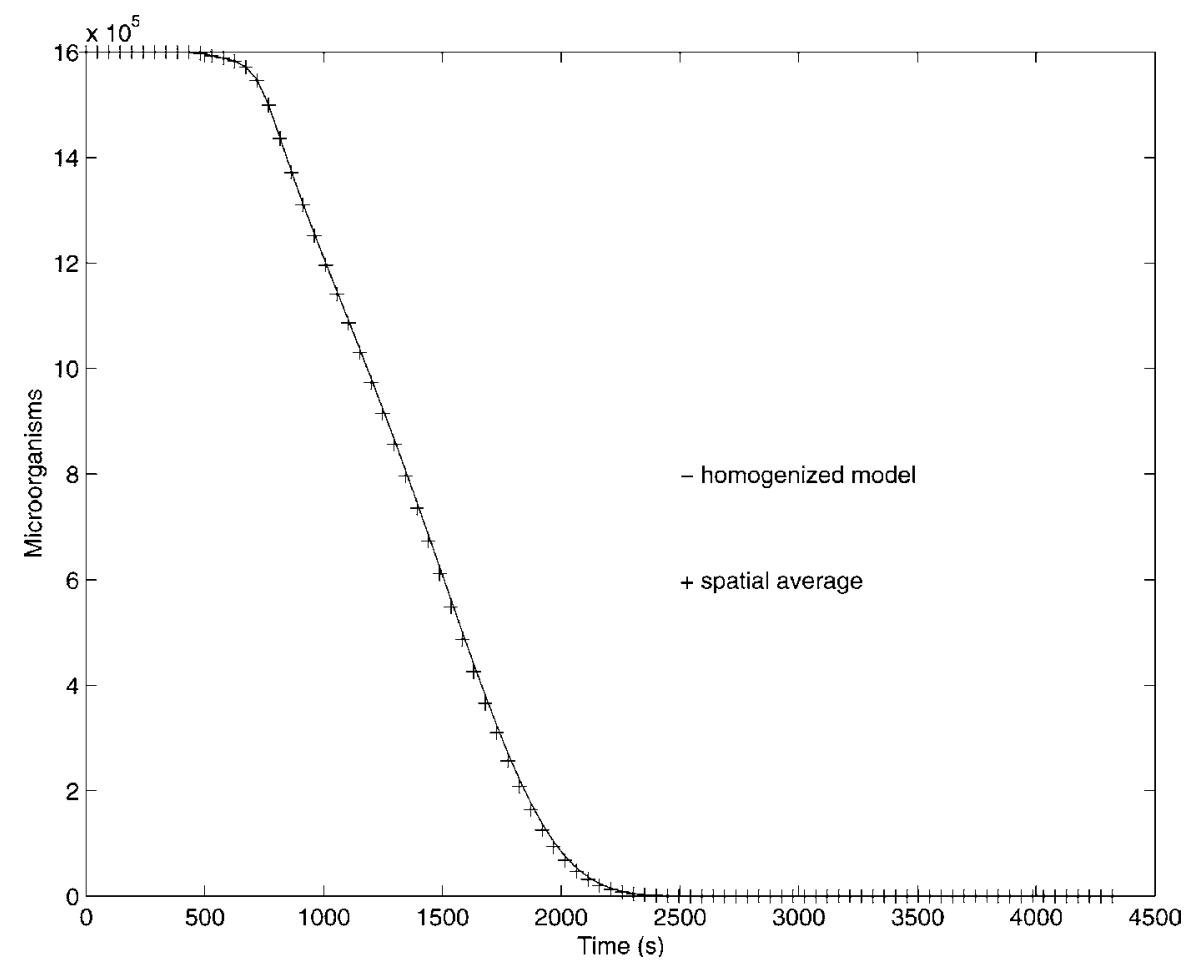

Fig. 7. Evolution in time of the numer of microorganims in the food pack.

\section{Conclusions}

In this work we have proved that the spatial average of the conductivity cannot be used in an homogenized heat transfer model, when the conductivity differences among the food components are high. We have pointed out that, in general, for industrial applications (where usually, the conductivity of the different food components are not very different) a thermal model using the spatial average of the conductivities can be applied without important deviations.

In a general case, we propose a spatial homogenisation procedure solving three steady state boundary value prob- 
lems, and to use the resulting equivalent conductivity tensor in the average heat transfer model. Although the heterogeneity in the food particles distribution leads to an anisotropic heat transfer behaviour, when the food particles are distributed at random, the deviation from the isotropic behaviour remains around the $1 \%$. A statistical average can be defined from a small number of possible particle arrangements, so that the deviations from the isotropic behaviour decrease to $0.1 \%$.

The modelling proposed in this paper can be applied to different heterogeneous thermal systems. This paper does not pretend to give general conclusions about the incidence of the number of food constituents, size and shape of the particles, food components conductivity ratio, spatial arrangement of food particles,.... This paper pretends to be a methodological approach to an accurate treatment of such systems. However, some preliminary conclusions have been attained, whose generality, at least from a qualitative point of view, seems to be right. The first conclusion is that for systems with food components conductivities of the same order, the spatial average and the equivalent conductivity are similar. This fact proves that most of industrial thermal predictions, based on the spatial average conductivity, are quite correct. An experimental procedure widely used in industries and laboratories consists in computing the equivalent conductivity from an inverse identification. Thus, the homogenized conduction thermal model is assumed as representing the heat transfer into the pack. Imposing a heating cycle and measuring the temperature evolution in one or several points into the pack, the equivalent conductivity is computed in order to fit the experimental results. This procedure, as most of experimental techniques available to measure the material conductivity (a good review can be found in the Rahman's book [10]), allows to identify an equivalent conductivity for homogeneous materials and mixtures. The numerical procedure proposed in this paper is an alternative technique that can be used to determine the equivalent conductivity of particulate systems, from the conductivity of each component, without additional experimental requirements.
We must point out again that the validity of the model requires convection effects small enough. This will be the case of systems containing a very viscous matrix, or very tortuous flow paths. In other cases, a slight error will be introduced, whose value will depend on the heat convection intensity.

\section{Acknowledgement}

This work has been supported by the Spanish research project PETRI 95-0135-OP-02.

\section{References}

[1] H. Carslaw, J. Jaeger, Conduction of Heat in Solids, Oxford Science Publications, 1959.

[2] J. Sanchez-Hubert, E. Sanchez-Palencia, Introduction aux Méthodes Asymptotiques et à l'Homogénéisation, Masson, Paris, 1992.

[3] M. Beran, Statistical Continuum Theories, Wiley, New York, 1968.

[4] M. Beran, Application of Statistical Theories for the Determination of Thermal, Electrical and Magnetic Properties of Heterogeneous Materials, Academic Press, New York, 1974.

[5] Z. Hashin, Theory of mechanical behaviour of heterogeneous media, Appl. Mech. Rev. 17 (1964) 1-9.

[6] Z. Hashin, Analysis of composite materials-A survey, J. Appl. Mech. 50 (1983) 481-505.

[7] M. Beran, J. McCoy, Mean field variations in a statistical sample of heterogeneous linearly elastic solids, Internat. J. Solids Structures 6 (1970) 1035-1054.

[8] P. Raviart, J. Thomas, Introduction à l'Analyse Numérique des Équations aux Dérivées Partielles, Masson, Paris, 1988.

[9] D.R. Heldman, D.B. Lund, Handbook of Food Engineering, Marcel Dekker, New York, 1992.

[10] S. Rahman, Food Properties Handbook, CRC, 1995.

[11] A. Mateu, Validación de procesos térmicos en continuo mediante el uso de un integrador tiempo temperatura (ITT) microbiológico y de modelos matemáticos, $\mathrm{PhD}$ Thesis, Universidad Politécnica de Valencia, Spain, 1997.

[12] A. Mateu, F. Chinesta, M. Ocio, M. García, A. Martínez, Development and validation of a mathematical model for HTST processing of foods containing large particles, J. Food Protection 60 (10) (1997) 12241229 